\title{
Ursolic acid sensitizes cisplatin-resistant HepG2/DDP cells to cisplatin via inhibiting Nrf2/ARE pathway
}

This article was published in the following Dove Press journal:

Drug Design, Development and Therapy

25 October 2016

Number of times this article has been viewed

\section{Shouhai $\mathrm{Wu}^{1,2}$ \\ Tianpeng Zhang' \\ Jingsheng $\mathrm{Du}^{3}$}

'School of Life Sciences, Sun Yat-sen University, ${ }^{2}$ Center for Regenerative and Translational Medicine, ${ }^{3}$ Department of Pharmacy, The Second Affiliated Hospital, Guangzhou University of Chinese Medicine, Guangzhou, Guangdong, People's Republic of China
Correspondence: Jingsheng Du Department of Pharmacy, The Second Affiliated Hospital, Guangzhou University of Chinese Medicine, No II I Dade Road, Yuexiu District, Guangzhou 510120, Guangdong, People's Republic of China Email dujingshenggz@yeah.net
Background: Combinations of adjuvant sensitizers with anticancer drugs is a promising new strategy to reverse chemoresistance. Ursolic acid (UA) is one of the natural pentacyclic triterpene compounds known to have many pharmacological characteristics such as anti-inflammatory and anticancer properties. This study investigates whether UA can sensitize hepatocellular carcinoma cells to cisplatin.

Materials and methods: Cells were transfected with nuclear factor erythroid-2-related factor 2 (Nrf2) small interfering RNA and Nrf2 complementary DNA by using Lipofectin 2000. The cytotoxicity of cells was investigated by Cell Counting Kit 8 assay. Cell apoptosis, cell cycle, reactive oxygen species, and mitochondrial membrane potential were detected by flow cytometry fluorescence-activated cell sorting. The protein level of Nrf2, NAD(P)H quinone oxidoreductase 1 (NQO1), glutathione $S$-transferase (GST), and heme oxygenase-1 (HO-1) was detected by Western blot analysis.

Results: The results showed that the reverse index was 2.9 - and 9.69 -fold by UA of $1.125 \mu \mathrm{g} / \mathrm{mL}$ and $2.25 \mu \mathrm{g} / \mathrm{mL}$, respectively, for cisplatin to HepG2/DDP cells. UA-cisplatin combination induced cell apoptosis and reactive oxygen species, blocked the cell cycle in G0/G1 phase, and reduced the mitochondrial membrane potential. Mechanistically, UA-cisplatin dramatically decreased the expression of Nrf2 and its downstream genes. The sensibilization of UA-cisplatin combination was diminished in Nrf2 small interfering RNA-transfected HepG2/DDP cells, as well as in Nrf2 complementary DNA-transfected HepG2/DDP cells.

Conclusion: The results confirmed the sensibilization of UA on HepG2/DDP cells to cisplatin, which was possibly mediated via the Nrf2/antioxidant response element pathway.

Keywords: ursolic acid, chemoresistance, cisplatin, liver cancer, Nrf2/ARE

\section{Introduction}

Hepatocellular carcinoma is one of the most common malignant tumors worldwide. ${ }^{1}$ Chemotherapy is one of the important methods in the comprehensive treatment of liver cancer, and the effect of drugs on the treatment of liver cancer is particularly weighty. ${ }^{1}$ The combined chemotherapy based on cisplatin recommended by international cancer organizations has become a line of liver cancer standard chemotherapy regimens. ${ }^{1}$ With the widespread application of platinum drugs, the tumor cell has inevitably developed resistance to platinum, which significantly reduces the effect of chemotherapy. ${ }^{2}$ Earlier studies reported that the drug resistance of recurrent liver cancer increased significantly, while the response rate of chemotherapy drugs was $<30 \%$. $^{2}$ Thus, there is an emergent need to develop a new drug sensitizer that can increase the efficacy of cisplatin-based chemotherapy and overcome drug resistance. 
Nuclear factor erythroid-2-related factor 2 (Nrf2) is known as the "primary supervisor" of the antioxidant response through the antioxidant response elements (AREs), regulating the expression of numerous genes including heme oxygenase-1 (HO-1), NAD(P)H quinone oxidoreductase 1 (NQO1), and glutathione $S$-transferase (GST) and several adenosine triphosphate-dependent drug efflux pumps (multidrug resistance proteins)..$^{3-5}$ Therefore, many studies have demonstrated that $\mathrm{Nrf2}$ is a transcription factor that can regulate various cytoprotective genes. Recently, Nrf2 has been reported to be a pharmacological target to overcome drug resistance. Overexpression of Nrf2 increases chemoresistance; on the contrary, knockdown of Nrf2 sensitizes various cancer cells including liver, ${ }^{6}$ leukemia, ${ }^{7}$ neuroblastoma, ${ }^{8}$ lung, ${ }^{9}$ breast, ${ }^{10}$ and pancreatic ${ }^{11}$ cells to chemotherapeutic drugs. Hence, screening Nrf2 inhibitors as an adjuvant sensitizer to overcome drug resistance is a desirable treatment strategy.

Earlier studies reported that one of the potential drug targets of several pentacyclic triterpene compounds, such as maslinic acid, ${ }^{12}$ boswellic acid, ${ }^{13}$ and oleanolic acid, ${ }^{14}$ was Nrf2. This finding make us to assess whether other pentacyclic triterpene compounds can inhibit Nrf2/ARE pathway to reverse resistance to chemotherapy drugs. Ursolic acid (UA), a natural pentacyclic triterpene compound (Figure 1), which is widely found in medicinal plants such as holy basil, food, and other plants, exhibits anticancer potential effect in vitro and in vivo. ${ }^{15}$ It inhibited proliferation and caused apoptosis in cells of numerous cancers, including breast cancer, ${ }^{16}$ colon cancer, ${ }^{17}$ non-small cell lung cancer, ${ }^{18}$ cervical cancer, ${ }^{19}$ multiple myeloma, ${ }^{20}$ pancreatic cancer, ${ }^{21}$ melanoma, ${ }^{22}$ and prostate cancer. ${ }^{23}$ UA has been shown to prevent $\mathrm{CCl}_{4}$-induced hepatotoxicity and fibrosis via Nrf2/ARE pathway. ${ }^{24}$ In this study, we tested the effect of UA to sensitize cisplatin-resistant human hepatocarcinoma HepG2/DDP cells to cisplatin-induced

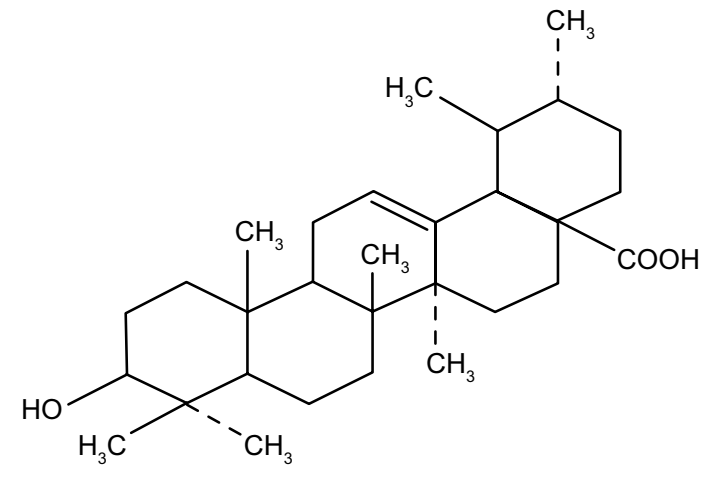

Figure I Chemical structure of UA Abbreviation: UA, ursolic acid. cytotoxicity and identified the underlying mechanism of its action. The results demonstrated that the combination of UA with low dose of cisplatin exhibited significantly higher cytotoxic response in HepG2/DDP cells. Mechanistically, UA-cisplatin combination significantly decreased the level of Nrf2 and its downstream genes, leading to a reversal of cisplatin-resistant phenotype in HepG2/DDP cells. Thus, UA is considered to be a promising adjuvant sensitizer to overcome chemoresistance.

\section{Materials and methods \\ Regents}

HepG2 cells were obtained from the American Type Culture Collection (ATCC, Manassas, VA, USA). HepG2/DDP cells were obtained from the Cell Bank, Chinese Academy of Sciences, Shanghai, People's Republic of China. Cisplatin, $\mathrm{UA}$, and dichloro-dihydro-fluorescein diacetate were purchased from Sigma-Aldrich Co. (St Louis, MO, USA). Cell Counting Kit 8 (CCK8), JC-1 dye, and BCA Protein Assay Kit were obtained from Beyotime (Nantong, People's Republic of China). Annexin V Fluorescein Isothiocyanate Apoptosis KGA107 Detection Kit was purchased from KeyGene (Nanjing, People's Republic of China). Dulbecco's Modified Eagle's Medium and fetal bovine serum were obtained from Thermo Fisher Scientific (Waltham, MA, USA). Polyvinylidene difluoride (PVDF) membrane was obtained from EMD Millipore (Billerica, MA, USA). Anti-Nrf2, anti-HO-1, anti-NQO1, anti-GST, anti-glyceraldehyde-3-phosphate dehydrogenase (GAPDH) antibody, and horseradish-peroxidase-conjugated secondary antibody were obtained from Santa Cruz Biotechnology Inc. (Dallas, TX, USA).

\section{Cell culture}

HepG2 cells were maintained in Dulbecco's Modified Eagle's Medium supplemented with $10 \%$ fetal bovine serum and $1 \%$ penicillin/streptomycin at $37^{\circ} \mathrm{C}$ in a humidified $5 \%$ $\mathrm{CO}_{2}$ atmosphere. HepG2/DDP cells were maintained in the same medium and then treated with $0.03 \mu \mathrm{M}$ cisplatin for at least 4 weeks before the experiment. ${ }^{25}$

\section{Cell proliferation assay}

Cell proliferation was detected using the CCK8 according to the manufacturer's protocol. The cells were seeded at $1 \times 10^{3}$ per well in a 96-well plate and treated as experiment design. Then, $10 \mu \mathrm{L}$ of CCK 8 reagent was added to each well and incubated for 4 hours at $37^{\circ} \mathrm{C}$. VMax ${ }^{\circledR}$ microplate spectrophotometer (Molecular Devices LLC, Sunnyvale, CA, USA) was used to measure the absorbance at $490 \mathrm{~nm}$. 


\section{Cell apoptosis assay}

The Annexin-V Fluorescein Isothiocyanate Apoptosis KGA107 Detection Kit for flow cytometry fluorescenceactivated cell sorting (FACS; BD Biosciences, San Jose, CA, USA) was used. In this protocol, $5 \times 10^{5}$ cells were seeded and washed twice with phosphate-buffered saline, and then mixed with $500 \mu \mathrm{L}$ binding buffer to form a cell suspension. Annexin-V Fluorescein Isothiocyanate $5 \mu \mathrm{L}$ was mixed with $5 \mu \mathrm{L}$ of propidium iodide. The cells were incubated for 10 minutes at room temperature in the dark and analyzed immediately using an FACS.

\section{Cell cycle analysis}

The cells were plated at $2 \times 10^{5}$ per well in a six-well plate. After 24 hours, cells were harvested and fixed with ice cold $70 \%$ ethanol at $-4^{\circ} \mathrm{C}$ overnight. Then, the cells were incubated with $10 \mathrm{mg} / \mathrm{mL}$ RNase A, $400 \mathrm{mg} / \mathrm{mL}$ propidium iodide, and $0.1 \%$ Triton- $X$ in phosphate-buffered saline at room temperature for 30 minutes and analyzed immediately using an FACS.

\section{Measurement of reactive oxygen species}

According to manufactures' protocol, the level of reactive oxygen species (ROS) was measured by dichloro-dihydrofluorescein diacetate. Briefly, cells were incubated with dichloro-dihydro-fluorescein diacetate (final concentration of $10 \mu \mathrm{M}$ ) at room temperature in the dark for 30 minutes, then washed with cold Hank's balanced salt solution ( $\mathrm{pH}$ 7.2), and then analyzed immediately using a FACS.

\section{Measurement of mitochondrial membrane potential}

According to the manufacturer's instruction, mitochondrial membrane potential (MMP) was determined using JC-1 dye. Briefly, the cells $\left(5 \times 10^{4}\right.$ per well) were plated in six-well plates. After treatment, cells were collected and mixed with $500 \mathrm{~mL}$ JC-1 working solution at room temperature in the dark for 15-20 minutes. Then, the cells were resuspended in $500 \mu \mathrm{L} 1 \times$ incubation buffer, and analyzed immediately using a FACS.

\section{Western blotting}

The cell lysates were harvested in lysis buffer. Total protein content was analyzed using the BCA Protein Assay Kit. Samples were separated by $10 \%$ sodium dodecyl sulfate-polyacrylamide gel electrophoresis and electrotransferred to the PVDF membrane. The PVDF membrane was incubated with $10 \%$ nonfat dried milk in Tris-buffered saline (TBS; 100 mM NaCl, 10 mM Tris-HCl, pH 7.4) containing
$0.1 \%$ Tween 20 (TBST) for 2 hours. Overnight incubation was performed with anti-Nrf2 (diluted 1:2,000), anti-HO-1 (diluted 1:2,000), anti-NQO1 $(1: 2,000)$, anti-GST (diluted 1:2,000), or anti-GAPDH antibody (diluted $1: 10,000$ ) at $4{ }^{\circ} \mathrm{C}$, followed by incubation with horseradish-peroxidase-conjugated secondary antibodies. After rinsing in Tris-buffered saline containing $0.1 \%$ Tween 20 , the PVDF membrane was exposed to an X-ray film using Western blot detection reagents (Thermo Fisher Scientific). Gel band density was scanned using the Gel Doc $2000^{\circledR}$ system and analyzed by Quantity One ${ }^{\circledR}$ image software, version 5.2.1 (Bio-Rad Laboratories Inc., Hercules, CA, USA).

\section{Transient transfection with Nrf2 small interfering RNA or plasmids}

The small interfering RNA (siRNA) targeting human Nrf2 (5'-GAGUUACAGUGUCUUAAUA-3'), the nontargeting negative control siRNA (5'-UUCUCCGAACG UGUCACGUTT-3'), the plasmid pGL3-Nrf2, and empty vector DNA were obtained from Shanghai GenePharma (Shanghai, People's Republic of China). Cells were plated at a density of $1.5 \times 10^{5}$ cells/well in six-well plates. After 24 hours, cells were transfected with siRNA or plasmid mixed with Lipofectamine 2000 (Thermo Fisher Scientific).

\section{Statistical analysis}

All the data were expressed as mean \pm standard deviation. The statistical analysis was performed using one-way analysis of variance or two-tailed Student's $t$-test for multiple comparisons. The differences between comparisons were considered to be statistically significant at $P<0.05$. SPSS software version 18.0 (SPSS Inc., Chicago, IL, USA) was used for data analysis. All experiments were performed in triplicate.

\section{Results \\ Nrf2 was overexpressed in cisplatin- resistant human hepatocellular carcinoma HepG2/DDP cells}

Cell survival rates were detected by CCK 8 assay to observe the cisplatin resistance phenotype of cells exposed to cisplatin. HepG2 cells were treated with series concentration of cisplatin $(0.1-25.6 \mu \mathrm{g} / \mathrm{mL})$ for 48 hours (Figure 2A), and HepG2/DDP cells were treated with series concentration of cisplatin $(2-512 \mu \mathrm{g} / \mathrm{mL})$ for 48 hours (Figure 2B). Then, the inhibition rate of the cells was calculated. The results revealed that the $50 \%$ inhibitory concentration $\left(\mathrm{IC}_{50}\right)$ of HepG2/DDP cells was higher than that of HepG2 cells. 

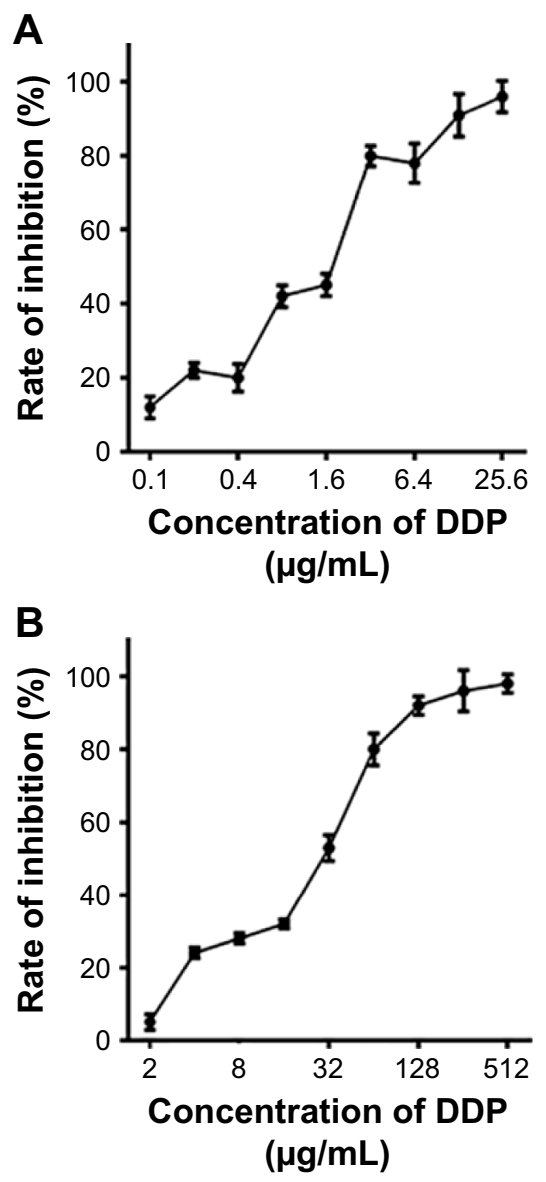

C

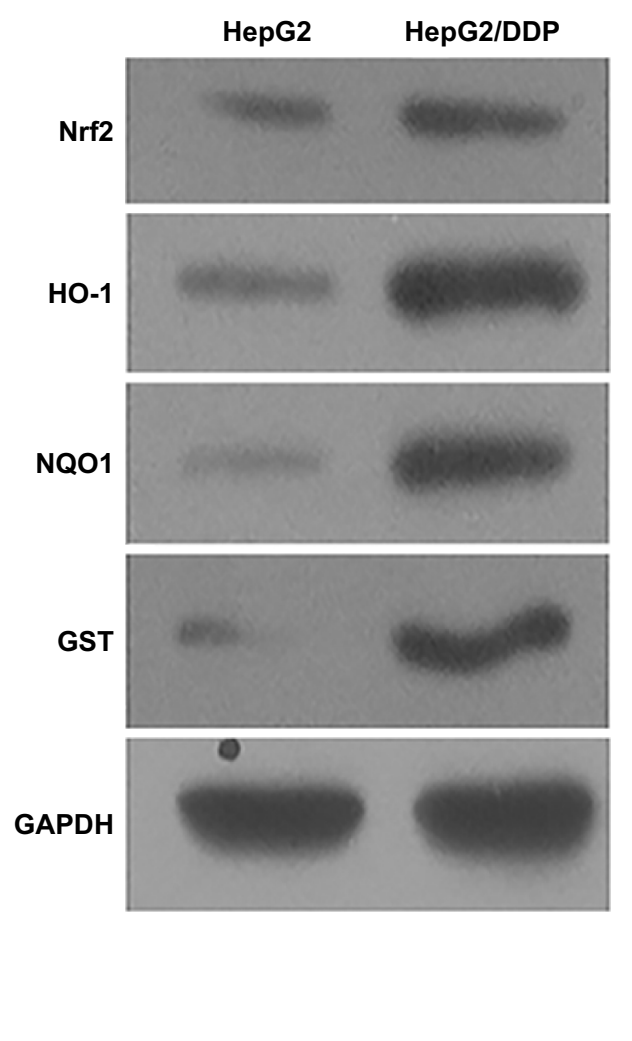

Figure $2 \mathrm{Nrf2}$ was overexpressed in cisplatin-resistant human hepatocellular carcinoma HepG2/DDP cells.

Notes: (A) HepG2 cells were treated with series concentration of cisplatin $(0.1-25.6 \mu \mathrm{g} / \mathrm{mL})$ for 48 hours. (B) HepG2/DDP cells were treated with series concentration of cisplatin (2-512 $\mu \mathrm{g} / \mathrm{mL})$ for 48 hours. (C) The level of Nrf2 and its downstream target genes HO-I, NQOI, and GST in HepG2 and HepG2/DDP cells was detected by Western blot assay. Results are representative of three different experiments, and they are expressed as mean \pm SD.

Abbreviations: GAPDH, glyceraldehyde-3-phosphate dehydrogenase; GST, glutathione S-transferase; HepG2, hepatocellular carcinoma cell line; HepG2/DDP, cisplatinresistant hepatocellular carcinoma cell line; HO-I, heme oxygenase-I; NQOI, NAD(P)H quinone oxidoreductase I; Nrf2, nuclear factor erythroid-2-related factor 2; $\mathrm{SD}$, standard deviation.

To evaluate whether Nrf2 and its substrates were different between HepG2 and HepG2/DDP cells, Western blot assay was performed. The level of Nrf2 and its downstream target genes HO-1, NQO1, and GST in HepG2/DDP cells was significantly higher than that in HepG2 cells (Figure 2C).

\section{UA potentiates cisplatin-induced growth inhibition}

As shown in Figure 3A, HepG2 and HepG2/DDP cells were treated with increasing concentrations of UA (1.125-288 $\mu \mathrm{g} / \mathrm{mL})$ for 48 hours. Then, the inhibition rate of the cells was calculated. It was found that the proliferation of HepG2 and HepG2/DDP cells was inhibited by UA after 48-hour incubation. We chose $1.125 \mu \mathrm{g} / \mathrm{mL}$ and $2.25 \mu \mathrm{g} / \mathrm{mL}$ of UA for the subsequent study as low cytotoxicity occurred at these two concentrations, thus excluding the anti-proliferation effect of high-dose UA in cancer cells.
To investigate whether UA can increase the sensitivity of HepG2/DDP cells to cisplatin, HepG2 cells were treated with series concentration of cisplatin $(0.1-25.6 \mu \mathrm{g} / \mathrm{mL})$ and/or UA (1.125 $\mu \mathrm{g} / \mathrm{mL}$ and $2.25 \mu \mathrm{g} / \mathrm{mL}$ ) for 48 hours (Figure $3 \mathrm{~B}$ ), and HepG2/DDP cells were treated with series concentration of cisplatin $(2-512 \mu \mathrm{g} / \mathrm{mL})$ and/or UA $(1.125 \mu \mathrm{g} / \mathrm{mL}$ and $2.25 \mu \mathrm{g} / \mathrm{mL}$ ) for 48 hours (Figure 3C). Then, $\mathrm{IC}_{50}$ was calculated. The results showed that the reversal index was 2.9- and 9.69-fold by UA of $1.125 \mu \mathrm{g} / \mathrm{mL}$ and $2.25 \mu \mathrm{g} / \mathrm{mL}$, respectively, for cisplatin (Figure 3D).

\section{UA-cisplatin combination enhances low- dose cisplatin-induced apoptosis and causes G0/GI arrest in resistant cells}

HepG2/DDP cells were treated with dimethyl sulfoxide as control group (final concentration of $0.1 \%$ ). HepG2/DDP cells were treated with $8.92 \mu \mathrm{g} / \mathrm{mL}$ cisplatin $(30 \%$ inhibitory 

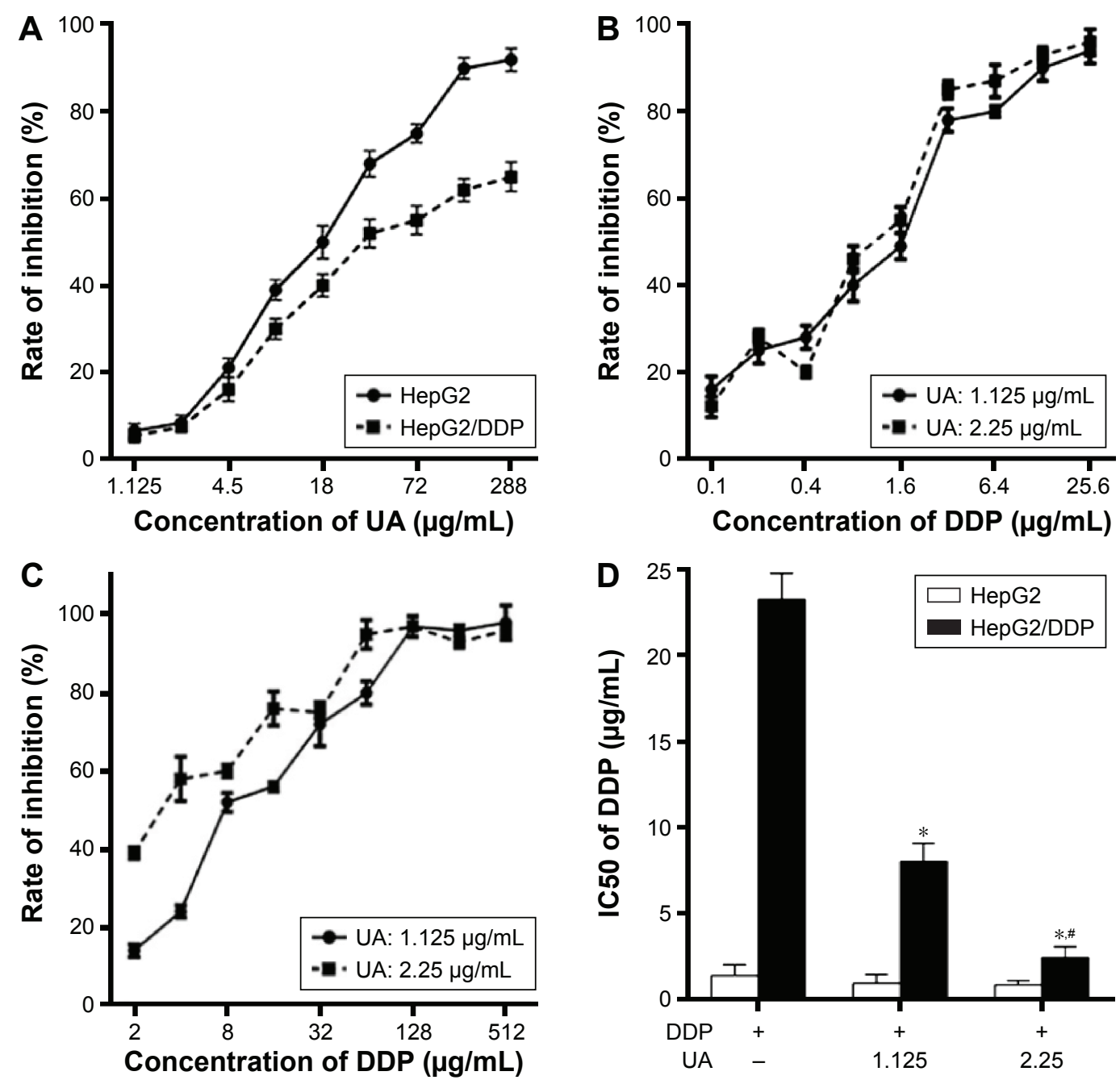

Figure 3 UA potentiates cisplatin-induced growth inhibition.

Notes: (A) HepG2 cells and HepG2/DDP cells were treated with increasing concentrations of UA (1.125-288 $\mu \mathrm{g} / \mathrm{mL}$ ) for 48 hours. (B) HepG2 cells were treated with series concentration of cisplatin $(0.1-25.6 \mu \mathrm{g} / \mathrm{mL})$ and UA $(1.125 \mu \mathrm{g} / \mathrm{mL}$ and $2.25 \mu \mathrm{g} / \mathrm{mL})$ for 48 hours. (C) HepG2/DDP cells were treated with series concentration of cisplatin $(2-512 \mu \mathrm{g} / \mathrm{mL})$ and UA (1.125 $\mu \mathrm{g} / \mathrm{mL}$ and $2.25 \mu \mathrm{g} / \mathrm{mL})$ for 48 hours. (D) HepG2 cells were treated for 48 hours with series concentration of cisplatin $(0.1-25.6 \mu \mathrm{g} / \mathrm{mL})$ and/or UA ( $(.125 \mu \mathrm{g} / \mathrm{mL}$ and $2.25 \mu \mathrm{g} / \mathrm{mL})$. HepG2/DDP cells were incubated for 48 hours with series concentration of cisplatin $(2-512 \mu \mathrm{g} / \mathrm{mL})$ and/or UA (1.125 $\mu \mathrm{g} / \mathrm{mL}$ and $2.25 \mu \mathrm{g} / \mathrm{mL}$ ). Then, $I C_{50}$ of cisplatin was calculated. $* P<0.05$ compared the HepG2/DDP cells alone treated with cisplatin. ${ }^{\#}<0.05$ vs the HepG2/DDP cells cotreated with cisplatin $(2-512 \mu \mathrm{g} / \mathrm{mL})$ and $\mathrm{UA}(1.125 \mu \mathrm{g} / \mathrm{mL})$. Results are representative of three different experiments, and they are expressed as mean \pm SD. The statistical analysis was performed using either one-way analysis of variance or two-tailed Student's $t$-test for multiple comparisons.

Abbreviations: HepG2, hepatocellular carcinoma cell line; HepG2/DDP, cisplatin-resistant hepatocellular carcinoma cell line; IC ${ }_{50}, 50 \%$ inhibitory concentration; SD, standard deviation; UA, ursolic acid.

concentration $\left[\mathrm{IC}_{30}\right]$ of cisplatin for HepG2/DDP cells) and/or UA $(2.25 \mu \mathrm{g} / \mathrm{mL})$ for 48 hours. The results showed that the proportion of apoptotic cells in the groups treated with UA and low-dose cisplatin combination was increased when compared to the groups treated with low-dose cisplatin $(P<0.01$; Figure 4A). As shown in Figure 4B, the groups treated with $\mathrm{UA}$ and low-dose cisplatin combination enhanced the cells in $\mathrm{G} 0 / \mathrm{G} 1$ phase, and reduced the number of cells in $\mathrm{G} 2 / \mathrm{M}$ phases $(P<0.05)$. These results demonstrate that UA increased the sensitivity of HepG2/DDP cells to low-dose cisplatin by retaining cells in G0/G1 phase and promoting cell apoptosis.

\section{UA-cisplatin combination increases low- dose cisplatin-induced mitochondrial oxidative stress in resistant cells}

To explore the sensibilization of UA on HepG2/DDP cells to low-dose cisplatin, the level of ROS and MMP was detected. The results showed that the level of ROS in the groups treated with UA and low-dose cisplatin combination was increased when compared to the groups treated with cisplatin $(P<0.01$; Figure 5A). In addition, we used JC-1 dye and the flow cytometry system to analyze the MMP (Figure 5B). The results showed that cells with low MMP in the groups 


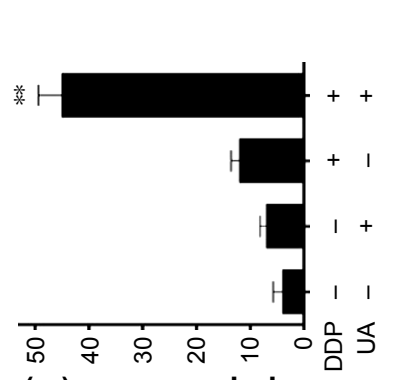

(\%) әүеג s!soldod $\forall$
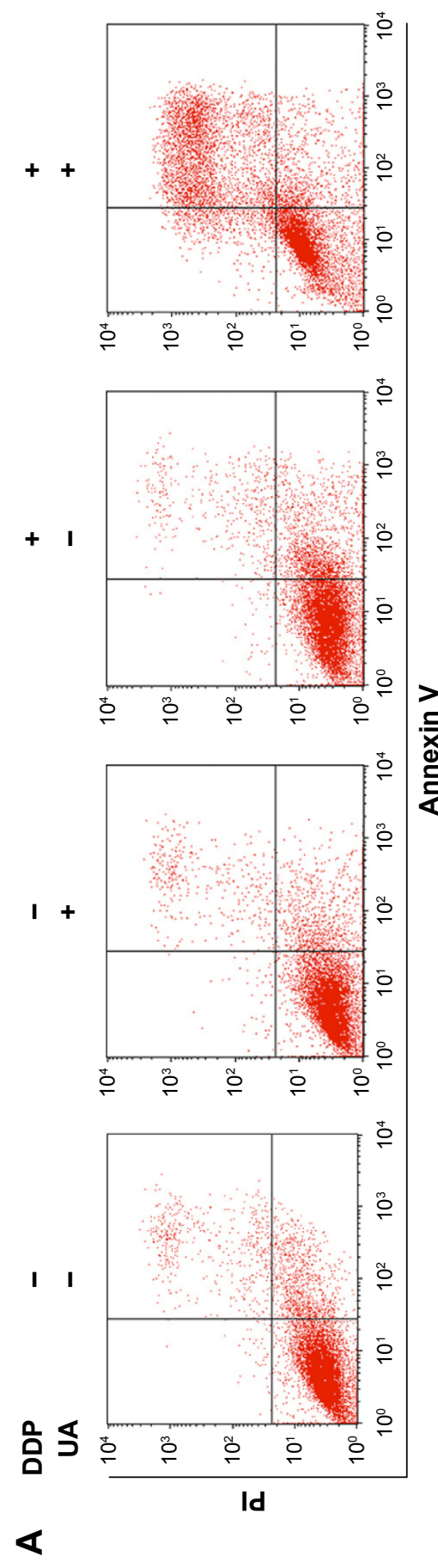
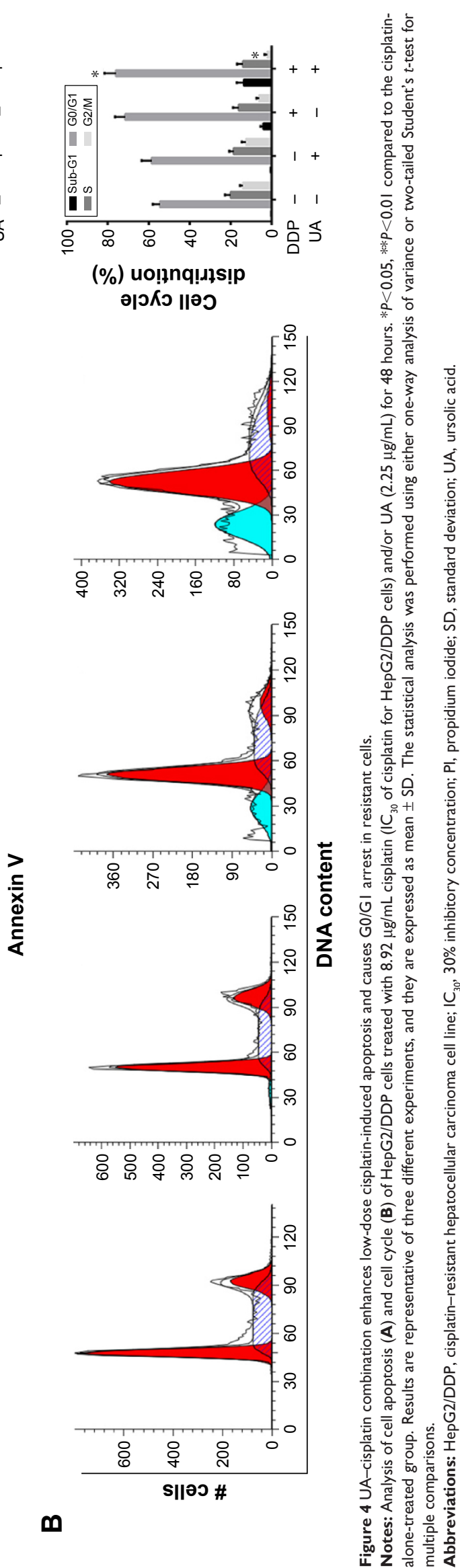


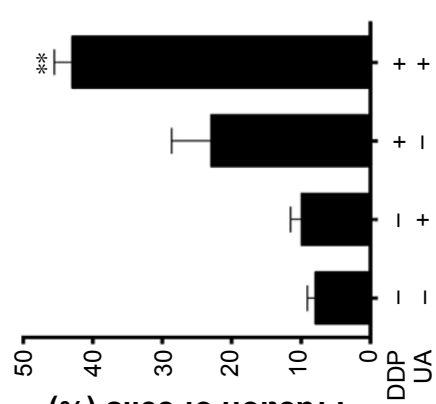

(\%) s||ә0 yo uo!łoed]
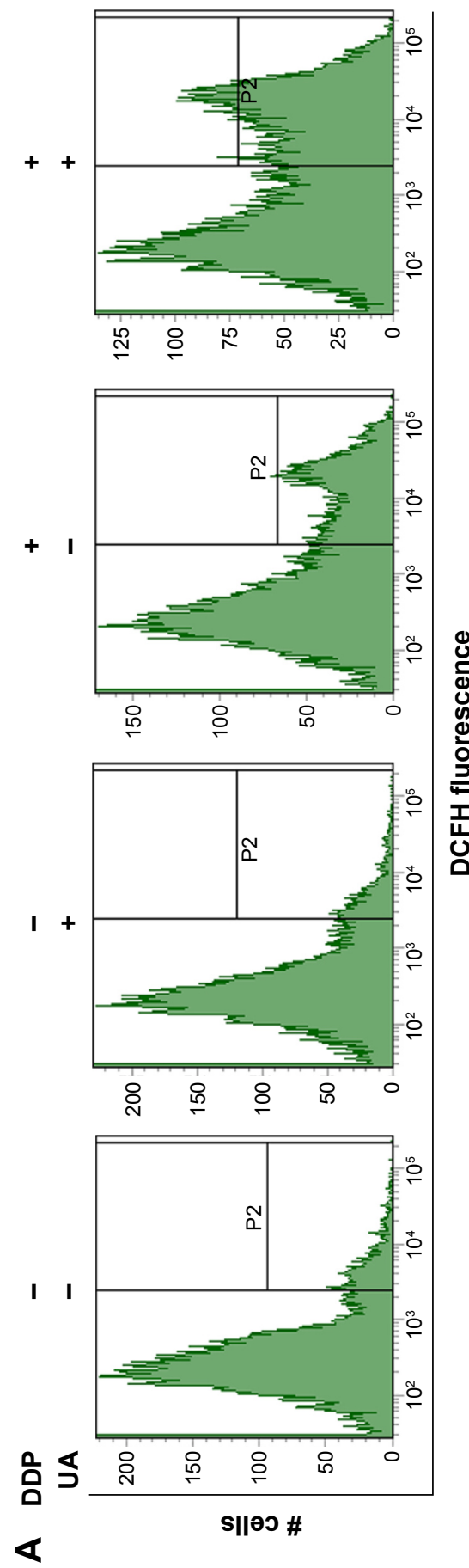

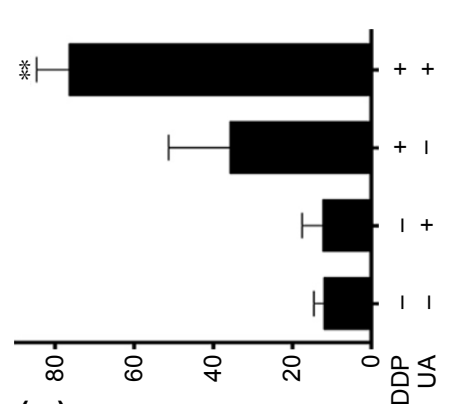

(\%) dWW Mо। पฺ!M s||әว
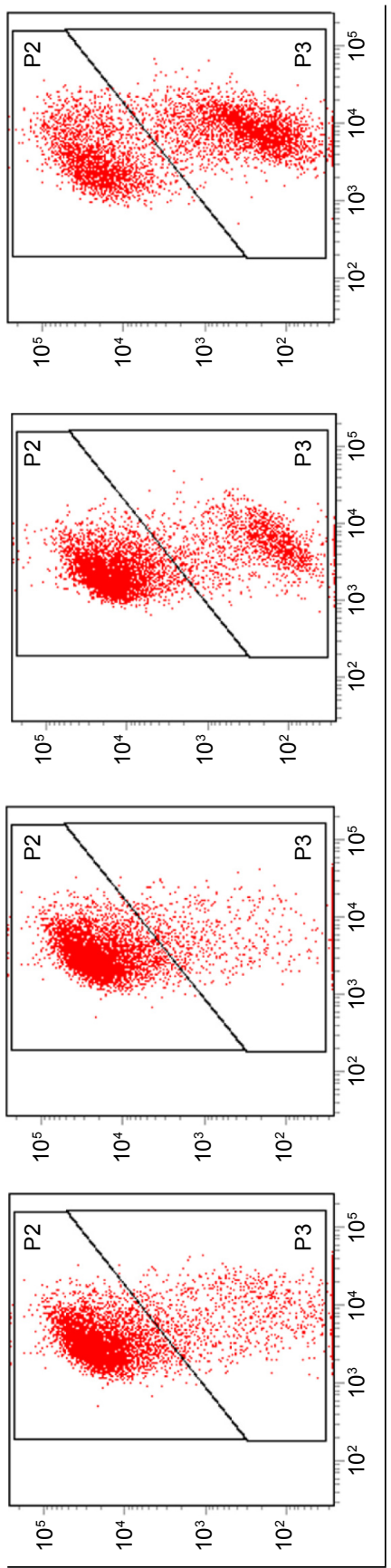

m

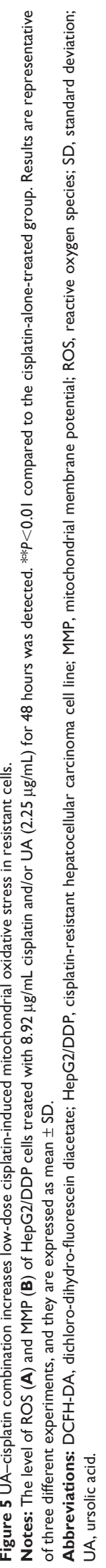


of HepG2/DDP cells treated with UA and low-dose cisplatin combination increased markedly compared to the groups treated with low-dose cisplatin $(P<0.01)$.

\section{UA-cisplatin combination downregulates Nrf2 and its substrates}

To investigate the regulation mechanism of the sensibilization of UA on HepG2/DDP cells to low-dose cisplatin, the protein expression levels of Nrf2, HO-1, NQO1, and GST were detected by Western blot analysis. As shown in Figure 6, the expression levels of Nrf2, HO-1, NQO1, and GST were decreased in the groups treated with UA and lowdose cisplatin combination compared with the groups treated with low-dose cisplatin.

\section{UA sensitizes HepG2/DDP cells to low- dose cisplatin via inhibition of Nrf2/ARE signaling pathway}

To investigate the potential involvement of Nrf2/ARE signaling pathway, HepG2/DDP cells were transfected with Nrf2 siRNA (si-Nrf2) or Nrf2 complementary DNA (Nrf2 cDNA) and then treated with $8.92 \mu \mathrm{g} / \mathrm{mL}$ cisplatin $\left(\mathrm{IC}_{30}\right.$ of cisplatin for HepG2/DDP cells) and/or UA $(2.25 \mu \mathrm{g} / \mathrm{mL})$ for 48 hours. The level of Nrf2, HO-1, NQO1, and GST was detected by Western blot analysis. As shown in Figure 7A, when compared to negative control (si-Con) transfected groups, the protein level of Nrf2, HO-1, NQO1, and GST was successfully downregulated in si-Nrf2 transfected groups.

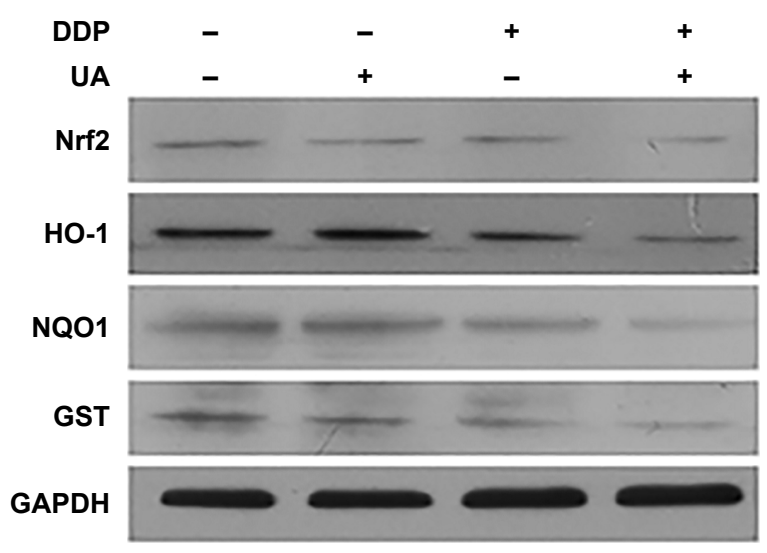

Figure 6 UA-cisplatin combination downregulates $\mathrm{Nrf2}$ and its substrates. Notes: The protein expression levels of Nrf2, HO-I, NQOI, and GST of HepG2/ DDP cells treated with $8.92 \mu \mathrm{g} / \mathrm{mL}$ cisplatin and/or UA $(2.25 \mu \mathrm{g} / \mathrm{mL})$ for 48 hours were detected by Western blot analysis. Results are representative of three different experiments, and they are expressed as mean \pm SD.

Abbreviations: GAPDH, glyceraldehyde-3-phosphate dehydrogenase; GST, glutathione S-transferase; HepG2/DDP, cisplatin-resistant hepatocellular carcinoma cell line; HO-I, heme oxygenase-I; NQOI, NAD(P)H quinone oxidoreductase I; Nrf2, nuclear factor erythroid-2-related factor 2; SD, standard deviation; UA, ursolic acid.
Furthermore, UA and low-dose cisplatin combination did not significantly influence the level of Nrf2, HO-1, NQO1, and GST in si-Nrf2-transfected HepG2/DDP cells. As shown in Figure $7 \mathrm{C}$, when compared to empty vector (vector) transfected groups, the protein level of Nrf2, HO-1, NQO1, and GST was successfully upregulated in Nrf2 cDNA-transfected groups. Furthermore, UA and low-dose cisplatin combination significantly decreased the protein expression of $\mathrm{Nrf}$, HO-1, NQO1, and GST in Nrf2 cDNA-transfected HepG2/ DDP cells.

Then, we observed the sensibilization of UA to cisplatin in HepG2/DDP cells with Nrf2 knockdown or overexpression. HepG2/DDP cells were transfected with si-Nrf2 or Nrf2 cDNA, and then treated with series concentration of cisplatin $(2-512 \mu \mathrm{g} / \mathrm{mL})$ and/or UA $(2.25 \mu \mathrm{g} / \mathrm{mL})$ for 48 hours. Transfection with si-Nrf2 in HepG2/DDP cells was much more sensitive to cisplatin than in HepG2/ DDP cells (Figure 7B). Figure 7D shows that Nrf2 cDNA transfection did not significantly affect the chemoresistance of HepG2/DDP cells. However, the sensibilization of UA-cisplatin combination was diminished in si-Nrf2transfected HepG2/DDP cells (Figure 7B), as well as in Nrf2 cDNA-transfected HepG2/DDP cells (Figure 7D).

\section{Discussion}

Drug resistance is one of the main hurdles to effective therapy of numerous tumors, which is taken into account during chemotherapy. ${ }^{2}$ Even a small increase in chemotherapy drug can cause severe toxicity to dose-limiting normal tissue. ${ }^{2}$ Thus, to accomplish higher curability with least toxicity during chemotherapy, strategies using dual agents rather than using single agents represent the most useful alternative. Various natural products have been considered as potential sources of chemosensitizer. Gao et $\mathrm{al}^{26}$ reported that "apigenin sensitized doxorubicin-resistance BEL-7402/ADM cells to doxorubicin". Chian et $\mathrm{al}^{27}$ discovered that "luteolin sensitized non-small cell lung cancer cell lines and colorectal cancer cells to oxaliplatin, bleomycin, and doxorubicin". Hou et $\mathrm{al}^{9}$ found that " $3{ }^{\prime}, 4^{\prime}, 5^{\prime}, 5,7$ pentamethoxyflavone sensitized cisplatin-resistant lung carcinoma A549 cells to cisplatin". UA is one of the natural pentacyclic triterpene compounds. It has numerous pharmacological activities including antioxidant, anti-inflammatory, antirheumatic, antiviral, and anticancer properties. In this study, we investigated the potential of UA to sensitize the cisplatin-resistant human hepatocellular carcinoma cells to cisplatin-induced cytotoxicity. We demonstrated that UA reduces the recalcitrance of HepG2/DDP cells to low-dose 
A

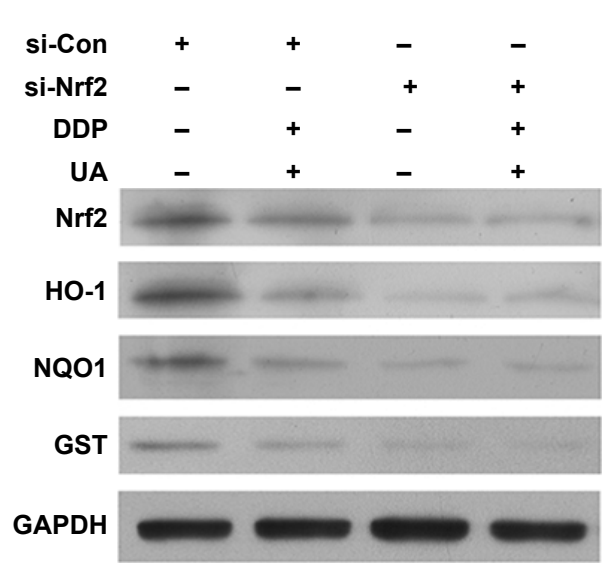

C

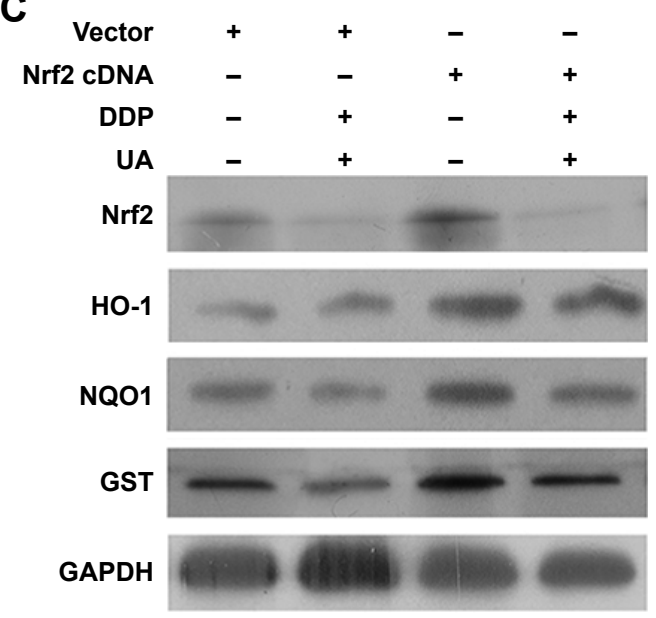

B

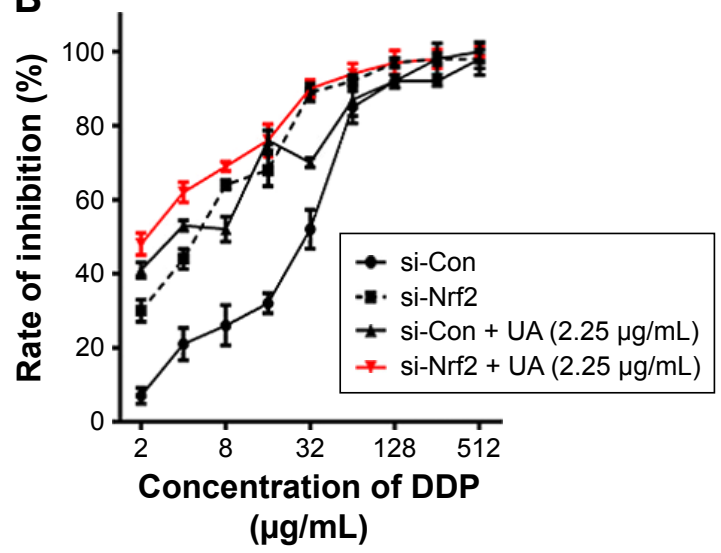

D

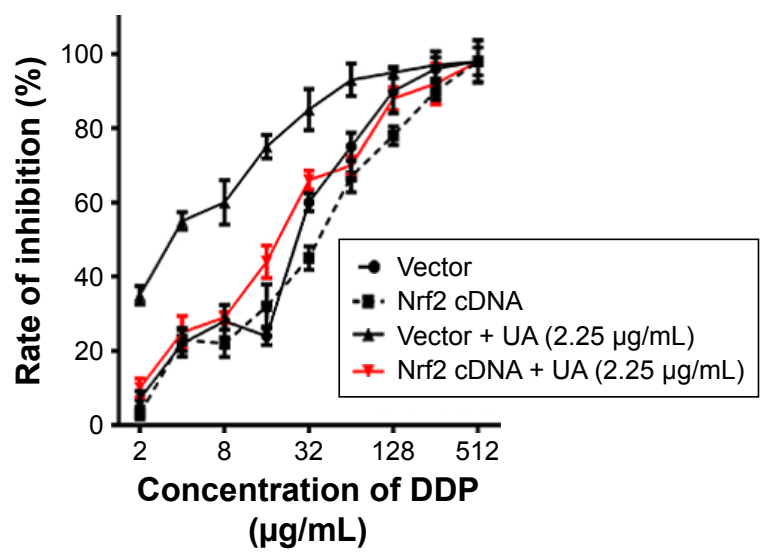

Figure 7 UA sensitizes HepG2/DDP cells to low-dose cisplatin via inhibition of Nrf2/ARE signaling pathway.

Notes: (A) HepG2/DDP cells were transfected with Nrf2 siRNA (si-Nrf2) or negative control (si-Con), or (C) HepG2/DDP cells were transfected with Nrf2 cDNA or empty vector (Vector), then treated with $8.92 \mu \mathrm{g} / \mathrm{mL}$ cisplatin (IC ${ }_{30}$ of cisplatin for HepG2/DDP cells) and/or UA (2.25 $\left.\mu \mathrm{g} / \mathrm{mL}\right)$ for $48 \mathrm{hours}$. The level of Nrf2, HO-I, NQOI, and GST was detected by Western blot analysis. (B) HepG2/DDP cells were transfected with si-Nrf2 or si-Con, or (D) HepG2/DDP cells were transfected with Nrf2 cDNA or empty vector (Vector), then treated with series concentration of cisplatin (2-5/2 $\mu \mathrm{g} / \mathrm{mL})$ and/or UA $(2.25 \mu \mathrm{g} / \mathrm{mL})$ for $48 \mathrm{hours}$. The inhibition rate of cell was detected by CCK8 assay. Results are representative of three different experiments, and they are expressed as mean \pm SD.

Abbreviations: ARE, antioxidant response element; CCK8, Cell Counting Kit 8; cDNA, complementary DNA; GAPDH, glyceraldehyde-3-phosphate dehydrogenase; GST, glutathione S-transferase; HepG2/DDP, cisplatin-resistant hepatocellular carcinoma cell line; HO-I, heme oxygenase-I; IC 30 , 30\% inhibitory concentration; NQOI, $\mathrm{NAD}(\mathrm{P}) \mathrm{H}$ quinone oxidoreductase I; Nrf2, nuclear factor erythroid-2-related factor 2; SD, standard deviation; siRNA, small interfering RNA; UA, ursolic acid.

cisplatin-induced cytotoxicity via downregulation of Nrf2/ARE signaling pathway.

The anticancer effect of cisplatin is:

binding to DNA and causing the DNA strands to crosslink, which ultimately triggers cells to die in a programmed way including the suppression of DNA synthesis, the restraint of RNA transcription, the blocking of cell cycle, and cell apoptosis. $^{28}$

Antiproliferative activity of UA which related to cell apoptosis and cell cycle arrest has been exhibited in several cancers. Mahmoudi et $\mathrm{al}^{29}$ reported that:

UA significantly suppressed cell proliferation after 24 and $48 \mathrm{~h}$ in the presence of UA, and increased apoptosis by enhancing the Annexin-V-positive population of isolated human melanoma cells.

Yang et $\mathrm{al}^{30}$ reported that "UA significantly arrested cycle in G0/G1 phase of hepatocellular carcinoma cells". However, the underlying mechanism of synergized antiproliferative effects of UA-cisplatin on cancer cells is not completely understood. We observed an increase in low-dose cisplatininduced apoptosis and an accumulation of G0/G1 cell population in UA-cisplatin-treated HepG2/DDP cisplatinresistant human hepatocellular carcinoma cells. These results imply that the combined treatment of low-dose UA and cisplatin manifests as the synergetic growth inhibitory effect on cisplatin-resistant cells. 
In the cytoplasm, cisplatin interacts with glutathione, metallothioneins, or mitochondrial proteins such as the voltagedependent anion channel, resulting in the generation of ROS; ROS can directly trigger membrane permeabilization, thereby triggering cisplatin-induced cell death; quenching proapoptotic ROS is known to be responsible for cisplatin resistance. ${ }^{28}$ Recent research has suggested that:

UA enhances radiation effects by increasing ROS,

downregulating Ki-67 level, and improving apoptosis in

BGC-823 human adenocarcinoma gastric cancer cells. ${ }^{31}$

Kim et $\mathrm{al}^{32}$ reported that UA induced apoptosis by decreasing MMP as shown with JC-1 staining in breast cancer cells. In our study, we observed that UA-cisplatin combination increased low-dose cisplatin-induced mitochondrial oxidative stress in resistant cells. Our findings suggested that an increase in ROS and a decrease in MMP are responsible for, at least in part, cisplatin resistance reduced by UA.

Nrf2 is known to be a pharmacological target to overcome drug resistance. ${ }^{33}$ In our study, the expression level of Nrf2 and its downstream target genes HO-1, NQO1, and GST in HepG2/DDP cells was significantly higher than that in HepG2 cells. The expression level of Nrf2-mediated pathway showed a decrease in Nrf2 siRNA-transfected HepG2/ DDP cells, which led to slight recovery of sensitivity to treatment with cisplatin. Our findings suggest a crucial role of Nrf2/ARE signaling pathway in the chemoresistance of human hepatocellular carcinoma cells. Therefore, the identification of potent small-molecule inhibitors of $\mathrm{Nrf} 2$ is desirable. Nrf2 is the potential drug target of several pentacyclic triterpene compounds, including UA. The study by Ma et $\mathrm{al}^{24}$ suggested that:

UA protected liver from carbon tetrachloride (CCL4)induced hepatotoxicity and liver fibrosis through Nrf2/ARE pathway in an experimental mice model.

Moreover, earlier studies showed that:

UA relieved cytotoxicity and the stimulation of external deleterious factors, reverted the intracellular redox balance in a cigarette-smoke-extract-induced human bronchial epithelial cell model; UA reduced cigarette-smoke-extractinduced DNA damage via the Nrf2 pathway. ${ }^{34}$

In this study, we confirmed UA as an available Nrf2 inhibitor. UA-cisplatin treatment sensitized HepG2/DDP cells to lowdose cisplatin and inhibited the level of Nrf2 and its substrates. However, in Nrf2 siRNA- or Nrf2 cDNA-transfected HepG2/DDP cells, the sensibilization of UA-cisplatin combination was diminished, indicating that suppression of Nrf2/ARE signaling pathway was one of the main mechanisms by which UA can weaken chemoresistance.

Many articles have reported that other signaling pathways also regulate the Nrf2/ARE pathway, including various transcription factors such as peroxisome proliferator-activated receptor, estrogen receptor $\alpha$, and activating transcription factor $3 .{ }^{35}$ Therefore, agents that downregulate Nrf2/ARE pathway might activate these transcription factors directly in cancer cells. Whether the regulatory mechanism of Nrf2/ ARE pathway by UA-cisplatin combination is related to these transcription factors has yet to be studied.

\section{Conclusion}

Our data experimentally showed the chemosensitization of UA on hepatocellular carcinoma cisplatin-resistant HepG2/ DDP cells to low-dose cisplatin via Nrf2/ARE pathway and suggested that UA as a possible natural adjuvant sensitizer might have clinical significance with therapeutic capability on overcoming cisplatin-resistant hepatocellular carcinoma cells. However, different hepatocellular carcinoma cells, or even more other cancer cells, should be used for further study to see whether it is a universal phenomenon, or it occurs only in this cell line. Recently, many researches have suggested that the overcoming cisplatin resistance by using different adjuvant molecules and nanoparticle technology, 36,37 the comparison between these new drugs and UA-cisplatin combination should be studied in the future. Further safety and efficacy investigations in animal experiment and clinical trials are essential before UA can be used as a chemosensitizer in the treatment of liver cancer.

\section{Acknowledgment}

The authors are thankful to the members of our laboratories for their insight and technical support.

\section{Disclosure}

The authors report no conflicts of interest in this work.

\section{References}

1. Buendia MA, Neuveut C. Hepatocellular carcinoma. Cold Spring Harb Perspect Med. 2015;5(2):a021444.

2. Gordon RR, Nelson PS. Cellular senescence and cancer chemotherapy resistance. Drug Resist Updat. 2012;15(1-2):123-131.

3. Xu W, Shao L, Zhou C, Wang H, Guo J. Upregulation of Nrf2 expression in non-alcoholic fatty liver and steatohepatitis. Hepatogastroenterology. 2011;58(112):2077-2080.

4. Liu J, Wu KC, Lu YF, Ekuase E, Klaassen CD. Nrf2 protection against liver injury produced by various hepatotoxicants. Oxid Med Cell Longev. 2013;2013:305861.

5. Wu KC, Liu JJ, Klaassen CD. Nrf2 activation prevents cadmium-induced acute liver injury. Toxicol Appl Pharmacol. 2012;263(1):14-20. 
6. Guan L, Zhang L, Gong Z, et al. FoxO3 inactivation promotes human cholangiocarcinoma tumorigenesis and chemoresistance through Keap1-Nrf2 signaling. Hepatology. 2016;63(6):1914-1927.

7. Yao J, Wei X, Lu Y. Chaetominine reduces MRP1-mediated drug resistance via inhibiting PI3K/Akt/Nrf2 signaling pathway in K562/Adr human leukemia cells. Biochem Biophys Res Commun. 2016;473(4): 867-873.

8. Furfaro AL, Piras S, Domenicotti C, et al. Role of Nrf2, HO-1 and GSH in neuroblastoma cell resistance to bortezomib. PLoS One. 2016;11(3): e0152465

9. Hou X, Bai X, Gou X, et al. 3', 4',5',5,7-pentamethoxyflavone sensitizes Cisplatin-resistant A549 cells to Cisplatin by inhibition of Nrf2 pathway. Mol Cells. 2015;38(5):396-401.

10. Syu JP, Chi JT, Kung HN. Nrf2 is the key to chemotherapy resistance in MCF7 breast cancer cells under hypoxia. Oncotarget. 2016;7(12): 14659-14672.

11. Hong YB, Kang HJ, Kwon SY, et al. Nuclear factor (erythroid-derived 2)like 2 regulates drug resistance in pancreatic cancer cells. Pancreas 2010;39(4):463-472.

12. Qin X, Qiu C, Zhao L. Maslinic acid protects vascular smooth muscle cells from oxidative stress through Akt/Nrf2/HO-1 pathway. $\mathrm{Mol}$ Cell Biochem. 2014;390(1-2):61-67.

13. Ding Y, Chen M, Wang M, et al. Neuroprotection by acetyl-11-ketobeta-Boswellic acid, in ischemic brain injury involves the Nrf2/HO-1 defense pathway. Sci Rep. 2014;4:7002.

14. Chen P, Zeng H, Wang Y, et al. Low dose of oleanolic acid protects against lithocholic acid-induced cholestasis in mice: potential involvement of nuclear factor-E2-related factor 2-mediated upregulation of multidrug resistance-associated proteins. Drug Metab Dispos. 2014;42(5):844-852.

15. Wozniak L, Skapska S, Marszalek K. Ursolic acid - a pentacyclic triterpenoid with a wide spectrum of pharmacological activities. Molecules. 2015;20(11):20614-20641.

16. Zhao C, Yin S, Dong Y, et al. Autophagy-dependent EIF2AK3 activation compromises ursolic acid-induced apoptosis through upregulation of MCL1 in MCF-7 human breast cancer cells. Autophagy. 2013;9(2):196-207.

17. Kim JH, Kim YH, Song GY, et al. Ursolic acid and its natural derivative corosolic acid suppress the proliferation of APC-mutated colon cancer cells through promotion of beta-catenin degradation. Food Chem Toxicol. 2014;67:87-95.

18. Wu J, Zhao S, Tang Q, et al. Activation of SAPK/JNK mediated the inhibition and reciprocal interaction of DNA methyltransferase 1 and EZH2 by ursolic acid in human lung cancer cells. J Exp Clin Cancer Res. 2015;34:99.

19. Leng S, Hao Y, Du D, et al. Ursolic acid promotes cancer cell death by inducing Atg5-dependent autophagy. Int J Cancer. 2013;133(12): 2781-2790.

20. Pathak AK, Bhutani M, Nair AS, et al. Ursolic acid inhibits STAT3 activation pathway leading to suppression of proliferation and chemosensitization of human multiple myeloma cells. Mol Cancer Res. 2007; 5(9):943-955.

21. Leal AS, Wang R, Salvador JA, Jing Y. Synthesis of novel ursolic acid heterocyclic derivatives with improved abilities of antiproliferation and induction of p53, p21waf1 and NOXA in pancreatic cancer cells. Bioorg Med Chem. 2012;20(19):5774-5786.
22. Junco JJ, Mancha-Ramirez A, Malik G, et al. Ursolic acid and resveratrol synergize with chloroquine to reduce melanoma cell viability. Melanoma Res. 2015;25(2):103-112.

23. Meng Y, Lin ZM, Ge N, Zhang DL, Huang J, Kong F. Ursolic acid induces apoptosis of prostate cancer cells via the PI3K/Akt $/ \mathrm{mTOR}$ pathway. Am J Chin Med. 2015;43(7):1471-1486.

24. Ma JQ, Ding J, Zhang L, Liu CM. Protective effects of ursolic acid in an experimental model of liver fibrosis through Nrf2/ARE pathway. Clin Res Hepatol Gastroenterol. 2015;39(2):188-197.

25. Wang XB, Wang SS, Zhang QF, et al. Inhibition of tetramethylpyrazine on P-gp, MRP2, MRP3 and MRP5 in multidrug resistant human hepatocellular carcinoma cells. Oncol Rep. 2010;23(1):211-215.

26. Gao AM, Ke ZP, Wang JN, Yang JY, Chen SY, Chen H. Apigenin sensitizes doxorubicin-resistant hepatocellular carcinoma BEL-7402/ ADM cells to doxorubicin via inhibiting PI3K/Akt/Nrf2 pathway. Carcinogenesis. 2013;34(8):1806-1814.

27. Chian S, Li YY, Wang XJ, Tang XW. Luteolin sensitizes two oxaliplatinresistant colorectal cancer cell lines to chemotherapeutic drugs via inhibition of the Nrf2 pathway. Asian Pac J Cancer Prev. 2014;15(6): 2911-2916.

28. Galluzzi L, Senovilla L, Vitale I, et al. Molecular mechanisms of cisplatin resistance. Oncogene. 2012;31(15):1869-1883.

29. Mahmoudi M, Rabe SZ, Balali-Mood M, Karimi G, Tabasi N, RiahiZanjani B. Ursolic acid induced apoptotic cell death following activation of caspases in isolated human melanoma cells. Cell Biol Int. 2015;39(2):230-236.

30. Yang LJ, Tang Q, Wu J, et al. Inter-regulation of IGFBP1 and FOXO3a unveils novel mechanism in ursolic acid-inhibited growth of hepatocellular carcinoma cells. J Exp Clin Cancer Res. 2016;35(1):59.

31. Yang Y, Jiang M, Hu J, et al. Enhancement of radiation effects by ursolic acid in BGC-823 human adenocarcinoma gastric cancer cell line. PLoS One. 2015;10(7):e0133169.

32. Kim KH, Seo HS, Choi HS, Choi I, Shin YC, Ko SG. Induction of apoptotic cell death by ursolic acid through mitochondrial death pathway and extrinsic death receptor pathway in MDA-MB-231 cells. Arch Pharm Res. 2011;34(8):1363-1372.

33. Taguchi K, Motohashi H, Yamamoto M. Molecular mechanisms of the Keap1-Nrf2 pathway in stress response and cancer evolution. Genes Cells. 2011;16(2):123-140.

34. Liu W, Tan X, Shu L, et al. Ursolic acid inhibits cigarette smoke extract-induced human bronchial epithelial cell injury and prevents development of lung cancer. Molecules. 2012;17(8):9104-9115.

35. Hayes JD, McMahon M. NRF2 and KEAP1 mutations: permanent activation of an adaptive response in cancer. Trends Biochem Sci. 2009;34(4):176-188.

36. Johnstone TC, Suntharalingam K, Lippard SJ. The next generation of platinum drugs: targeted $\mathrm{Pt}(\mathrm{II})$ agents, nanoparticle delivery, and $\mathrm{Pt}(\mathrm{IV})$ Prodrugs. Chem Rev. 2016;116(5):3436-3486.

37. Pathak RK, Dhar S. A nanoparticle cocktail: temporal release of predefined drug combinations. J Am Chem Soc. 2015;137(26):8324-8327.

\section{Publish your work in this journal}

Drug Design, Development and Therapy is an international, peerreviewed open-access journal that spans the spectrum of drug design and development through to clinical applications. Clinical outcomes, patient safety, and programs for the development and effective, safe, and sustained use of medicines are the features of the journal, which

\section{Dovepress}

has also been accepted for indexing on PubMed Central. The manuscript management system is completely online and includes a very quick and fair peer-review system, which is all easy to use. Visit http://www.dovepress.com/testimonials.php to read real quotes from published authors. 